www.nature.com/pj

\title{
Near-infrared fluorescent nanoparticle of low-bandgap $\pi$-conjugated polymer for in vivo molecular imaging
}

\author{
Hiroyuki Aoki ${ }^{1,2}$, Jun-ichi Kakuta ${ }^{1}$, Tomoyuki Yamaguchi ${ }^{1}$, Satoshi Nitahara ${ }^{1}$ and Shinzaburo Ito ${ }^{1,2}$
}

Polymer Journal (2011) 43, 937-940; doi:10.1038/pj.2011.86; published online 21 September 2011

Keywords: fluorescence resonance energy transfer; in vivo imaging; molecular probe; nanoparticle; near infrared fluorescence

\section{INTRODUCTION}

Molecular imaging, which visualizes biological molecules, cells and tissue, has recently attracted considerable attention. ${ }^{1-3}$ Various techniques, such as magnetic resonance imaging and positron emission tomography, have been developed in the past few decades. Among these techniques, the fluorescence imaging method is a useful modality for biological objects because the detection by fluorescence is highly sensitive, and because various kinds of information are available from the spectrum and temporal profile of the fluorescence signal. In the recent studies on molecular bioimaging probes and drug delivery systems, nanoparticles have received considerable attention because of their applicability in various imaging modalities and as drug carriers in in vivo systems. ${ }^{4-6}$ The site specificity of the nanoparticles and their lifetime in the blood circulation system are dependent on the size and the surface properties of the particles. ${ }^{7}$ Particles with a size of approximately $100 \mathrm{~nm}$ is known to be accumulated in tumor tissues by the enhanced permeability and retention (EPR) effect. ${ }^{8-10}$ Moreover, the incorporation of one or more chemicals in a single particle and the particle's surface modification could result in the multi-functional molecular probe. The nanoemulsion method with polymer materials is one of the most versatile technique, because the particle size can be precisely controlled in the range of $10 \mathrm{~nm}$ or greater; the physical and chemical properties are adjustable via the matrix polymer materials, and various lowmolecular-weight chemicals can be embedded in the particles. Therefore, the polymer nanoparticles (PNPs) that contain a fluorescent moiety are promising systems for the molecular imaging probes. The following requirements should be met for the quantitative optical in vivo imaging by a fluorescent molecular probe: (1) a large absorption coefficient for the excitation light, (2) a high quantum efficiency of fluorescence emission and (3) an excitation/emission wavelength range in the near-infrared (NIR) region. To achieve high signal intensity, the molecular probe should efficiently absorb photons. This absorption could be attained by loading dye moieties into a particle at high concentration. Not only the absorption efficiency but also the fluorescence quantum yield is an important factor. The NIR region of $600-900 \mathrm{~nm}$ is the spectral window for the optical imaging of living tissues, where the absorption and scattering are relatively low; ${ }^{11,12}$ therefore, the NIR excitation and emission enables the detection of fluorescence from deep inside of a living body. Numerous probes that operate in the NIR range, such as quantum dots, have been already reported. ${ }^{13,14}$ Here, we report the preparation of the PNPs that consist of a fluorescent $\pi$-conjugated polymer. Lowbandgap conjugated polymers developed in recent years have the absorption and emission bands at the wavelengths greater than $600 \mathrm{~nm} .^{15,16}$ We fabricated the PNPs using co-poly(2,3-diphenylthieno[3,4-b]pyrazine-alt-9,9-dioctylfluorene) $(\mathrm{CDPDOF})^{17}$ as a fluorescent molecular probe with a large absorption coefficient. Moreover, the emission quantum yield was enhanced by the fluorescent resonance energy transfer (FRET) mechanism. The application of the PNPs for the in vivo imaging of small animals is demonstrated.

\section{EXPERIMENTAL PROCEDURE}

A fluorescent-conjugated polymer CDPDOF (Figure 1a) was prepared by the Suzuki coupling reaction of 2,3-diphenylthieno[3,4-b]pyrazine and 5,7-dibromo-2,3-diphenylthieno[3,4-b]pyrazine, which were synthesized according to the previously published method. ${ }^{17}$ The weight- and number-average molecular weights, $M_{\mathrm{w}}$ and $M_{\mathrm{n}}$, were 18600 and 9940 , respectively. These values were determined by size-exclusion chromatography calibrated with polystyrene standards. Fluorescent-dye-doped PNPs were prepared by a nanoemulsion method. ${ }^{18-20}$ CDPDOF (400 $\left.\mu \mathrm{g}\right)$ and silicon 2,3-naphthalocyanine bis(trihexylsilyloxide) (SiNc, 16-200 $\mu$ g, Figure $1 \mathrm{~b}$ ) were dissolved in $0.8 \mathrm{ml}$ of chloroform. The polymer/dye solution was added to $10 \mathrm{ml}$ of an aqueous solution of polyoxyethylene sorbitan monolaurate (Tween 20, Tokyo Chemical Industry, Tokyo, Japan) at a concentration of $10 \mathrm{mg} \mathrm{ml}^{-1}$. After the solution was vigorously stirred for $10 \mathrm{~min}$, the nanoemulsion was prepared by the ultrasonication at a frequency of $22.5 \mathrm{kHz}$ and at a power of $20 \mathrm{~W}$ (Microson XL2000, Misonix, Farmingdale, NY, USA) for $30 \mathrm{~s}$ at a temperature of $0^{\circ} \mathrm{C}$. The surfactant-stabilized PNPs were obtained after the removal of chloroform by the heating and stirring of the sample solution. The ultraviolet-visible absorption and fluorescence spectra of the PNP dispersions were recorded with a UV-1800 spectrophotometer (Shimadzu, Kyoto, Japan) and a FP-6500

1Department of Polymer Chemistry, Kyoto University, Nishikyo, Kyoto, Japan and ${ }^{2}$ Advanced Biomedical Engineering Research Unit, Kyoto University, Nishikyo, Kyoto, Japan Correspondence: Dr H Aoki, Department of Polymer Chemistry, Kyoto University, Kyoto-Daigaku-Katsura, Nishikyo, Kyoto 615-8510, Japan. E-mail: aoki@photo.polym.kyoto-u.ac.jp

Received 9 May 2011; revised 18 July 2011; accepted 25 July 2011; published online 21 September 2011 
a

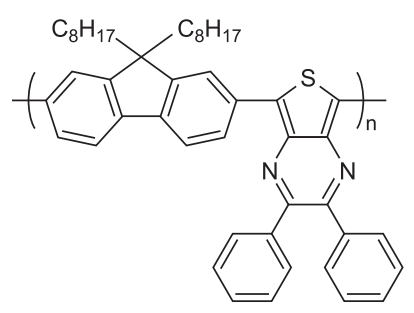

b

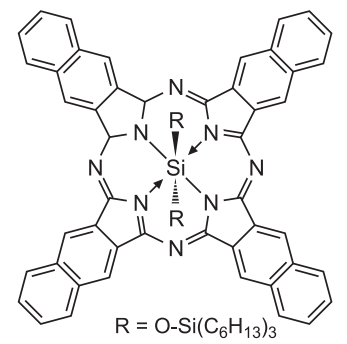

Figure 1 Chemical structures of co-poly(2,3-diphenylthieno[3,4-b]pyrazinealt-9,9-dioctylfluorene (CDPDOF) and silicon 2,3-naphthalocyanine bis(trihexylsilyloxide) ( $\mathrm{SiNc}$ ).

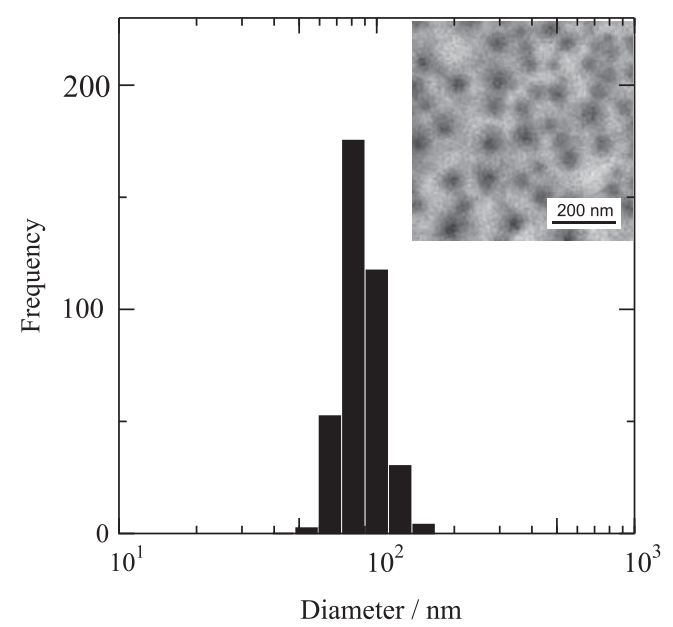

Figure 2 Size distribution for the polymer nanoparticle (PNP) of co-poly(2,3diphenylthieno[3,4-b]pyrazine-alt-9,9-dioctylfluorene (CDPDOF) observed by dynamic light scattering. The inset indicates the transmission electron microscope image.

spectrofluorometer (Jasco, Tokyo, Japan), respectively. The average particle sizes and size distributions were determined using the dynamic light scattering with the histogram method (ELS-Z, Otsuka Electronics, Osaka, Japan). Direct observation of the PNPs was conducted using a transmission electron microscope (JEM-2100F, JEOL, Tokyo, Japan). The PNPs were deposited on a copper grid covered with an elastic carbon support film. Transmission electron microscope imaging was performed without staining at a working voltage of $200 \mathrm{kV}$. In vivo fluorescence imaging of mice was performed using an IVIS-200 optical imaging system (Xenogen, Alameda, CA, USA). Normal nude mice (7 weeks old, male; BALB/c slc-nu/nu, Japan SLC, Hamamatsu, Japan) were intravenously injected with $200 \mu \mathrm{l}$ of PNP dispersed in phosphate-buffer saline (PBS). During the imaging, the mice were kept on the stage under anesthesia with isoflurane gas.

\section{RESULTS AND DISCUSSION}

Figure 2 shows the size distribution of the PNP of CDPDOF with SiNc (the fraction of SiNc was 1.0\%). This result indicates that the average diameter of the PNP was $82 \mathrm{~nm}$ with s.d. of $21 \mathrm{~nm}$. The size distribution of the PNP was not dependent on the SiNc fraction in the range of $0.4-50 \%$. Moreover, the size distribution was not altered after 30 days, indicating high dispersion stability in water. The transmission electron microscope image is shown in the inset of Figure 2 and indicates that the particle size and particle-size distribution were similar to the dynamic light scattering result. The size of the PNPs can be controlled in the range of $50-150 \mathrm{~nm}$ by adjusting the
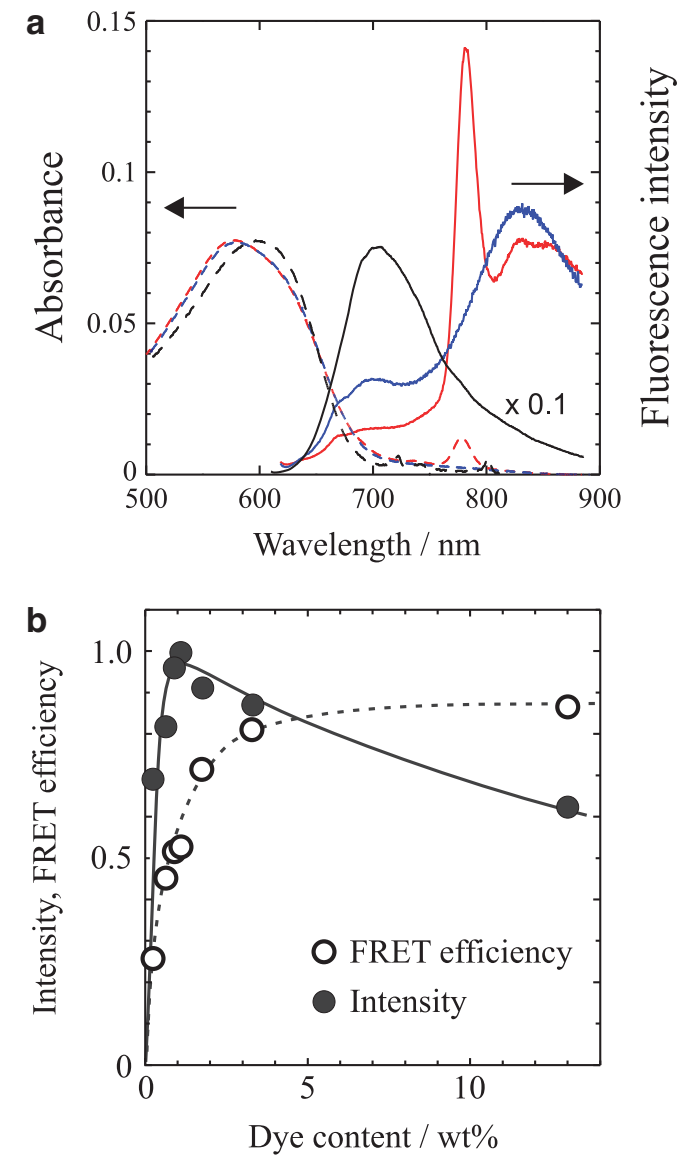

Figure 3 Absorption/fluorescence spectra for co-poly(2,3-diphenylthieno [3,4-b]pyrazine-alt-9,9-dioctylfluorene (CDPDOF) (a) and the dye content dependence of the fluorescence intensity/fluorescent resonance energy transfer (FRET) efficiency (b). In the panel a, the dashed and solid curves indicate the absorption and emission spectra, respectively. The black, blue and red curves indicate the spectra for a chloroform solution, CDPDOFpolymer nanoparticle (PNP), and CDPDOF/SiNc-PNP with a dye content of $0.15 \%$, respectively. In the panel $\mathbf{b}$, the open and filled circles indicate the fluorescence intensity and the FRET efficiency, respectively. The diameter of the PNP was $82 \mathrm{~nm}$. SiNc, silicon 2,3-naphthalocyanine bis(trihexylsilyloxide).

concentrations of the polymer and Tween 20 . The dispersion stability in PBS and fetal bovine serum solutions was examined. The PNPs were dispersed in PBS and fetal bovine serum (10\% in PBS) solutions at PNP concentrations of 0.05 and $0.45 \mathrm{mg} \mathrm{ml}^{-1}$, and the dispersion was kept at room temperature for one day. The dynamic light scattering and optical measurements showed no significant difference in the particle-size distribution or the absorption spectrum. These results indicate that the PNPs of CDPDOF can be homogeneously dispersed under in vivo conditions.

The ultraviolet-visible absorption and fluorescence spectra of a chloroform solution of CDPDOF and PNPs with and without SiNc are shown in Figure 3. Although the fluorescence spectra were observed under the excitation of CDPDOF at a wavelength of $600 \mathrm{~nm}$, the emission spectra were different from each other. The chloroform solution of CDPDOF emitted fluorescence at $690 \mathrm{~nm}$ with a quantum efficiency of 0.28 . The PNP of CDPDOF, however, showed another emission peak at $830 \mathrm{~nm}$ and a decreased intensity at the 690$\mathrm{nm}$ band (the blue curve in Figure 3a). The peak at $830 \mathrm{~nm}$ is due to 
inter-chain interactions among the conjugation system of CDPDOF. The $\pi$-conjugation is thought to be extended by stacking among the main chain of CDPDOF, resulting in the emission at a longer wavelength. Light-absorbing polymer particles are available by dispersing a low-molecular-weight dye; however, the dye fraction should be less than a few dozen percent to obtain PNPs with a narrow size distribution and high dispersion stability. The PNPs of CDPDOF consist only of the chromophoric monomer unit with a molar absorption coefficient of $4.4 \times 10^{4} \mathrm{M}^{-1} \mathrm{~cm}^{-1}$, resulting in a high absorption coefficient for the PNPs on the order of $10^{10} \mathrm{M}^{-1} \mathrm{~cm}^{-1}$. Although the PNP of the conjugated polymer absorbed the light with a high efficiency, the fluorescence quantum efficiency decreased to 0.02 because of the stacked chromophoric moieties. For the PNP of CDPDOF with SiNc, the fluorescence of SiNc was observed at $790 \mathrm{~nm}$, which resulted from FRET due to the large overlap of the emission and absorption bands of CDPDOF and SiNc, respectively. Despite the small fraction of SiNc $(0.15 \%)$, the peak intensity attributed to SiNc is larger than that of CDPDOF, indicating that the excitation energy of CDPDOF was efficiently converged to the SiNc moiety. The open symbols in Figure 3b show the energy transfer efficiency as a function of the dye content. The FRET efficiency increased with the increase of the dye content in the particle and reached a maximum value of about $85 \%$ at a concentration of $3 \%$. At low dye concentrations, the exciton is deactivated and emits fluorescence before reaching a SiNc molecule. For example, the average distance between the SiNc molecules is estimated to be $15 \mathrm{~nm}$ at a concentration of $0.15 \%$. This distance is relatively large compared with the diffusion length for conjugated polymers (on the order of $10 \mathrm{~nm}$ ). Therefore, a part of the exciton on CDPDOF cannot reach the SiNc, resulting in the low FRET efficiency of $26 \%$. The FRET efficiency increased with increasing the dye concentration in the PNP. The fluorescence intensity of SiNc decreased with the increase of the dye content larger than $1 \%$ (the filled circles in Figure 3b), whereas the FRET efficiency from CDPDOF to SiNc increased monotonically with the SiNc fraction up to a SiNc fraction of $3 \%$. This decrease is due to the self-quenching by the aggregation of SiNc. The fluorescence intensity at the optimum condition of $1.1 \%$ is 2.2 times larger than that of the CDPDOF-PNP without SiNc.

In the neat CDPDOF-PNP, the chromophoric monomer units were stacked with each other to form an excitation energy trap site, resulting in a reduction of the emission quantum yield. In contrast, in the PNPs of CDPDOF/SiNc, almost all the excitation energy was converged to the SiNc, and the PNPs emitted the fluorescence with relatively high quantum yield. Consequently, the emission intensity was improved by the FRET mechanism. The PNP of the low-bandgap polymer is compared with semiconductor quantum dots, which are used as fluorescent probes with a strong emission intensity. ${ }^{14}$ Quantum dots in NIR show a molar absorption coefficient of $0.5-5 \times 10^{6} \mathrm{M}^{-1} \mathrm{~cm}^{-1}$ and an emission quantum yield of 0.8 . Although the CDPDOF/SiNc-PNP showed a 20 -fold smaller quantum yield than that of the quantum dots, the absorption coefficient was several thousand times larger. Therefore, the emission intensity of the PNP is estimated to be larger by two orders of magnitude under the same excitation condition. Another advantage of the FRET mechanism is the tunability of the emission wavelength. The excitation and emission wavelengths are independently determined by the chemical structures of the energy-donating conjugated polymer material and the acceptor dye molecule, respectively. An appropriate selection of the dyes enables the multi-color imaging.

To consider the feasibility of the PNPs of the $\pi$-conjugated polymer as an in vivo imaging agent, whole bodies of mice were imaged. The mice were injected with the PBS dispersions of the PNPs of neat

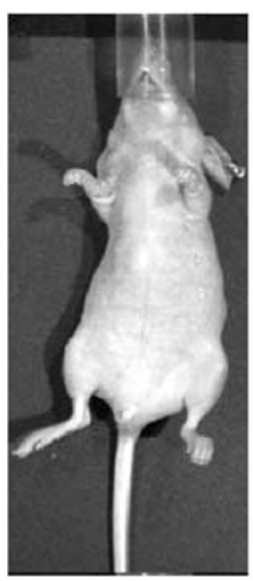

$0 \min$
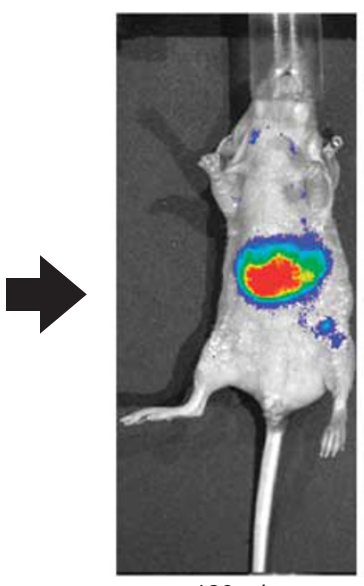

$180 \min$

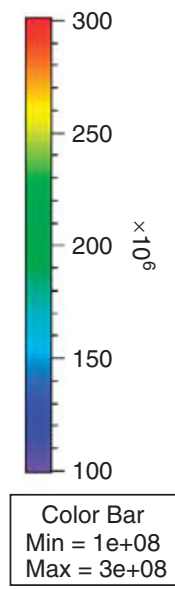

Figure 4 Images of a mouse injected by co-poly(2,3-diphenylthieno[3,4-b] pyrazine-alt-9,9-dioctylfluorene (CDPDOF)/silicon 2,3-naphthalocyanine bis (trihexylsilyloxide) (SiNc)-polymer nanoparticle (PNP). The left and right panels were acquired after 0 and 120 min of the injection, respectively. The fluorescence intensity from the PNP is indicated in color overlapped with the gray-scale image of the whole body of the mouse. The excitation and detection wavelengths were $605 \pm 15$ and $780 \pm 10 \mathrm{~nm}$, respectively.

CDPDOF and CDPDOF/SiNc with a diameter of $80 \mathrm{~nm}$. For the CDPDOF-PNP, no fluorescence signal was detected from the mouse body because the fluorescence intensity was weak. Furthermore, as shown in Figure 3a, the CDPDOF-PNP show a broad emission band in the NIR region longer than $850 \mathrm{~nm}$. The biological sample strongly absorbs the wavelengths longer than $900 \mathrm{~nm}$ because of the overtone of the vibration band of water. Therefore, the emission was partially absorbed by the tissue of the mouse. Moreover, the bandwidth of the emission holds practical importance in the fluorescence imaging. For the detection of the fluorescence from the CDPDOF-PNP, the light signal should be acquired over the broad wavelength range of $650-900 \mathrm{~nm}$; however, such a broadband detection is susceptible to a large noise floor due to the autofluorescence from the tissue. Thus, the fluorescence of the CDPDOF-PNP was not detectable. On the other hand, the signal from the CDPDOF/SiNc-PNP was clearly observed in the in vivo photoluminescence image shown in Figure 4, indicating that the PNP accumulated in the liver after 120 min after the injection. The bright emission at a NIR wavelength by an efficient energy-harvesting mechanism enabled the in vivo fluorescence imaging. The narrow bandwidth of the fluorescence from the energy acceptor dye moiety was effective in avoiding the background emission, which enables the quantitative measurement of the fluorescence intensity.

\section{SUMMARY}

Fluorescent nanoparticles at the NIR range were prepared from the low-bandgap conjugated polymer CDPDOF. The emission intensity was successfully enhanced by the FRET mechanism from CDPDOF to an energy-acceptor dye moiety, SiNc, resulting in the strong fluorescence in the NIR region. The fluorescence from the CDPDOF/SiNcPNP was brightly observed through a living body in in vivo optical imaging, indicating that the PNP accumulated in the liver of a mouse. The ability to target a specific site can be added by the surface modification of the PNPs with functional groups. The dye-doped PNPs of the low-bandgap polymer are thus a promising candidate for an in vivo molecular probe. 


\section{ACKNOWLEDGEMENTS}

We to thank Professor M Shimizu, Department of Material Chemistry, Kyoto University, for advice on the synthesis of CDPDOF, and Professor S Kondoh, Department of Biomolecular Engineering, Tokyo Institute of Technology, for the in vivo imaging of mice. This work is supported by The Innovative Techno-Hub for Integrated Medical Bio-imaging Project of the Special Coordination Funds for Promoting Science and Technology from the Ministry of Education, Culture, Sports, Science and Technology (MEXT).

1 Weissleder, R. \& Mahmood, U. Molecular imaging. Radiology 219, 316-333 (2001).

2 Massoud, T. F. \& Gambhir, S. S. Molecular imaging in living subjects: seeing fundamental biological processes in a new light. Gene Dev. 17, 545-580 (2003).

3 Weissleder, R. \& Pittet, M. J. Imaging in the era of molecular oncology. Nature 452, 580-589 (2008).

4 Sharma, P., Brown, S., Walter, G., Santra, S. \& Moudgil, B. Nanoparticles for bioimaging. Adv. Colloid Interface Sci. 123-126, 471-485 (2006).

5 Kingsley, J. D., Dou, H., Morehead, J., Rabinow, B., Gendelman, H. E. \& Destache, C. J. Nanotechnology: a focus on nanoparticles as a drug delivery system. J. Neuroimm. Pharm. 1, 340-350 (2006)

6 De Jong, W. H. \& Borm, P. J. A. Drug delivery and nanoparticles: applications and hazards. Int. J. Nanomed. 3, 133-149 (2008).

7 Moghimi, S. M., Hunter, A. C. \& Murray, J. C. Long-circulating and target-specific nanoparticles: theory to practice. Pharmacol. Rev. 53, 283-318 (2001).
8 Matsumura, Y. \& Maeda, H. A new concept for macromolecular therapeutics in cancer chemotherapy: mechanism of tumoritropic accumulation of proteins and the antitumor agent smancs. Cancer Res. 46, 6387-6392 (1986).

9 Maeda, H., Wu, J., Sawa, T., Matsumura, Y. \& Hori, K. Tumor vascular permeability and the epr effect in macromolecular therapeutics: a review. J. Control Release 65, 271-284 (2000).

10 Maeda, $\mathrm{H}$. The enhanced permeability and retention (epr) effect in tumor vasculature: the key role of tumor-selective macromolecular drug targeting. Adv. Enzyme Regul. 41, 189-207 (2001).

11 Weissleder, R. A clearer vision for in vivo imaging. Nat. Biotech. 19, 316-317 (2001).

12 Frangioni, J. V. In vivo near-infrared fluorescence imaging. Cur. Opin. Chem. Biol. 7, 626-634 (2003).

13 Amiot, C. A., Xu, S., Liang, S., Pan, L. \& Zhao, J. X. Near-infrared fluorescent materials for sensing of biological targets. Sensors 8, 3082-3105 (2008).

14 Gao, X., Yang, L., Petros, J. A., Marshall, F. F., Simons, J. W. \& Nie, S. In vivo molecular and cellular imaging with quantum dots. Cur. Opin. Biotech. 16,63-72 (2005).

15 van Mullekom, H. A. M., Vekemans, J. A. J. M., Havinga, E. E. \& Meijer, E. W. Developments in the chemistry and bandgap engineering of donor-acceptor substituted conjugated polymers. Mater. Sci. Eng. 32, 1-40 (2001).

16 Bundgaard, E. \& Krebs, F. C. Low bandgap polymers for organic photovoltaics. Solar Energy Mater. Solar Cells 91, 954-985 (2007).

17 Ashraf, R., Hoppe, H., Shahid, M., Gobsch, G., Sensfuss, S. \& Klemm, E. Synthesis and properties of fluorene-based polyheteroarylenes for photovoltaic devices. J. Polym. Sci. Part A: Polym. Chem. 44, 6952-6961 (2006).

18 Solans, C., Izquierdo, P., Nolla, J., Azemar, N. \& Garcia-Celma, M. J. Nano-emulsions. Cur. Opin. Colloid Interface Sci. 10, 102-110 (2005).

19 Anton, N., Benoit, J. \& Saulnier, P. Design and production of nanoparticles formulated from nano-emulsion templates-a review. J. Control Release 128, 185-199 (2008).

20 Landfester, K. Polyreactions in miniemulsions. Macromol. Rapid Commun. 22, 896-936 (2001). 